\title{
Experimental study of dynamic mechanical properties of rabbit knee ligaments
}

\author{
Sota Yamamoto ${ }^{1}$, Akinori Saito ${ }^{1}$, Masaki Kabayama ${ }^{2}$, Kei Nagasaka $^{1}$, \\ Koji Mizuno ${ }^{1}$ and Eiichi Tanaka ${ }^{1}$
}
1. Department of Mec hano-Informat ics and Systems, Nagoya University, Nagoya, Japan 2. Products Planning Heal thy Lif e Products Div., Home Appliances Company, Matsushita Ele ctric Works, Ltd., Hikone, Japan

\begin{abstract}
This study is concerned with the mechanical properties of knee joint ligaments, which are frequently injured in car-to-pedestrian collisions. Such injury makes a lot of disabilities in actions in daily life and it is difficult to recover the original mechanical functions of ligaments. Because of the lack of knowledge of the dynamic properties of these ligaments, there are some difficulties in finite element simulations of a car-to-pedestrian accident. Thus, we performed tensile tests on the rabbit medial collateral ligament (MCL) and anterior cruciate ligament (ACL) to evaluate the strain-rate dependence of their mechanical properties. The failure stress of the MCL increased with the strain rate. The avulsion load of the MCL tibial insertion was lower than the failure load of the MCL in all conditions tested. Three types of failure patterns were observed in the femur-ACL-tibia complex. The tangent modulus and the maximum stress of the ACL complex increased with the strain rate. The strain-rate dependence of the mechanical properties of knee ligaments was evaluated quantitatively. The results are expected to be useful for discussion of the injury criteria for the knee joint and its protection.
\end{abstract}

Key words: Medial collateral ligament, Anterior cruciate ligament, Mechanical property, Strain rate dependence

\section{INTRODUCTION}

Knee ligaments contribute to the stability of knee joint by controlling excessive motions. When the knee joints are injured in car-to-pedestrian collisions or sports accidents, the ligaments are frequently damaged because of the large external force exerted on the joints. Such injuries lead to many disabilities in actions in daily life and require a long time for healing and rehabilitation. Moreover, it is not easy to recover the original functions of knee ligament when they have been ruptured. Thus, to clarify the mechanism of knee injury is important for pedestrian protection and better QOL from the viewpoints of orthopaedics and injury prevention engineering. Recently, dynamic finite element analysis is coming into considerable use in studies of injury prevention, for example Takahashi et al. [1] However, a few data have been reported on the mechanical properties of knee joint ligaments at the high strain-rate found in car-to-pedestrian collisions [2, 3], however the strain-rate dependency of the mechanical properties of the ligament is not sufficiently understood. Therefore, the mechanical properties of knee ligaments must be elucidated for more accurate simulations.

In light of this background, we evaluated the strain-rate dependence of the mechanical properties of medial collateral ligament (MCL) and anterior cruciate ligament (ACL), which are frequently injured, in the rabbit. We perfomed tensile tests on the rabbit MCL at various strain rates and discussed the strain-rate dependence of mechanical properties of the substance and tibial insertion of the MCL. We also performed tensile tests on the femur-ACL-tibia complex and evaluated its failure.

\section{MATERIALS AND METHOD}

\subsection{Preparation for experiments}

The mechanical properties of the MCL were evaluated in ligament substance and tibial insertion of the MCL. The mechanical properties of the ligament substance were evaluated by tensile tests using femur-MCL complex specimens. The strength of tibial insertion of the MCL was evaluated using MCL-tibia complex specimens.

Femur-MCL complex specimens were obtained from 16 Japanese white rabbits (female, $2.8 \pm 0.17 \mathrm{~kg}$, mean \pm S.D.). The animals were sacrificed by injecting an overdose of pentobarbital sodium. The MCL was removed together with the distal femur and proximal tibia. Next, the femur-MCL-tibia complex was attached on a laser width meter type cross-sectional area meter [4], and its cross-sectional area was measured under $0.5 \mathrm{~N}$ of tension. The tibial insertion of MCL was then detached to avoid avulsion. The distal end of the MCL was fixed on a metal jig with cyanoacrylate cement. The femur was embedded in resin to make the femur easy to fix on the jig of tensile test machine. Two dot markers were added at a $5-\mathrm{mm}$ interval on the surface of the MCL substance using nygrosin to measure the MCL strain.

Corresponding author:

S. Yamamoto,sota@mech.nagoya-u.ac.jp 
MCL-tibia complex specimens were obtained from 13 Japanese white rabbits (female, $3.0 \pm 0.13 \mathrm{~kg}$, mean \pm S.D.) and prepared by a procedure similar to that for the femur-MCL complex specimens. The femoral insertion of the MCL was detached and the proximal end was fixed on a metal jig with cyanoacrylate cement. The tibia was embedded in resin. It is not easy to define the strain and at the tibial insertion because the insertion is attached on the flat part of the medial side of the proximal tibia. Thus, the nygrosin markers were omitted for the MCLtibia complex specimens.

During all preparation procedures described above, the MCL was kept in wet condition by appropriate dripapplication of physiological saline solution.

Seven Japanese white rabbits (female, $3.0 \pm 0.17 \mathrm{~kg}$, mean \pm S.D.) were used for ACL tests. Femur-ACL-tibia complex specimens were used for the ACL tensile tests. A femur-knee joint-tibia complex was removed and then attached on a plastic jig to define the geometry of the knee joint in the maximum extended position. Because of the twisted and inclined ACL structure, the geometry of the knee joint must be defined carefully. Woo et al. reported that the loading direction influences the failure properties of ACL [5], making the flexion angle of knee joint the most important geometrical parameter. Since our main focus is pedestrian protection, the human standing posture should be simulated in the experiments. The angle between the femur and tibia was examined in the maximally extended position to determine the set up of the knee joint geometry of the rabbit corresponding to the human standing posture. The joint angle was defined as 150 for the experimental set up of the femur-ACLtibia complex based on observation of sacrificed rabbits. The femur-knee joint-tibia complex was extended and fixed at a 150 joint angle on a plastic jig. Two line marks were then put on the frontal and lateral surfaces of the femur and tibia using nygrosin. These line markers were used as a reference to adjust the geometry of the knee joint when the specimen was chucked on the tensile test system. The surrounding soft tissue, the proximal femur and the distal tibia were then removed.

The cross-sectional area of the ACL midsection was measured in a non-twisted position by the measuring system [4] described above. The medial condyle and lateral condyle of femur were removed for the laser width meter measuring. The length of the ACL and width of its tibial insertion were also measured at the maximum joint angle by a vernier caliper.

Then the distal femur and proximal tibia were embedded in resin. Finally, two dot markers were added on the frontal edges of the femoral and tibial condyle using nygrosin to measure the strain of the ACL. The ACL was kept in a wet condition by appropriate dripapplication of physiological saline solution.

\subsection{Tensile tests}

A previously-developed dynamic/quasi-static tensile test system [4] was used. A schematic diagram of the system equipped with an $\mathrm{AC}$ servomotor and an $\mathrm{N}_{2}$ gas actuator is shown in Fig. 1. The velocity ranges of the servomotor and gas actuator are sequential, i.e., the servomotor works as the actuator for quasi-static tests when the elongation rate is between $0.01 \mathrm{~mm} / \mathrm{sec}$ and 10 $\mathrm{mm} / \mathrm{sec}$, and the $\mathrm{N}_{2}$ gas actuator is used for dynamic tests when the elongation rate is over $100 \mathrm{~mm} / \mathrm{sec}$. The force applied to a specimen was measured by a load cell on the piston.

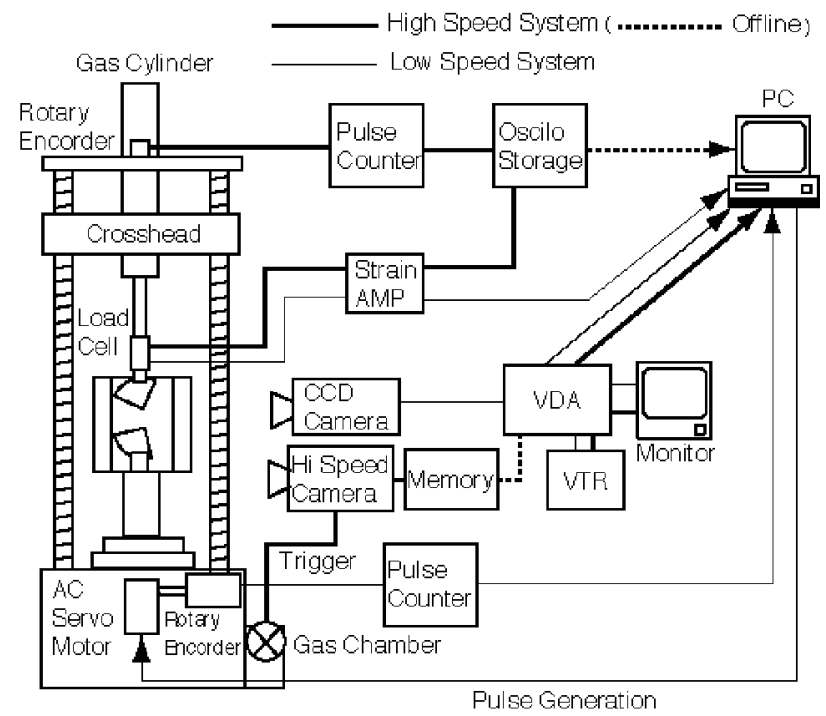

Fig. 1. Schematic diagram of tensile test system for MCL substance and tibial insertion.

The signals of the load cell and rotary encoder mounted on the piston are stored on a digital oscilloscope (Sony Techtronix, TDS420A). The relative displacement between two markers on ligament substance of the femur-MCL complex was recorded by a high-speed video camera (Kodak, HS-4540) for the dynamic tests or a CCD camera (Hamamatsu Photonics, C5405) for the quasi-static tests. The strain of the MCL substance was measured by a VDA (Hamamatsu Photonics, C3162). Both femur-MCL and MCL-tibia complexes were fixed on the test system in the position in which the longitudinal direction of MCL was aligned with the loading direction. The extension-rate conditions were set as $0.01,0.3$ and $10 \mathrm{~mm} / \mathrm{sec}$ for quasi-static tests and 100 and $300 \mathrm{~mm} / \mathrm{sec}$ for dynamic tests. All experiments were performed in $37^{\circ} \mathrm{C}$ physiological saline solution.

The same tensile test system used for the MCL experiments was employed for the ACL tensile test. Only the strain measuring method was changed. The relative displacement of the markers was recorded using a digital video camera recorder (DCR-VX2000, SONY) in quasistatic conditions and a digital high-speed video camera (MEMRECAM fx-K3, NAC Image Technology) in dynamic conditions, and analyzed using a video analyzing software (Movious Pro, MIRA). All of the tests were performed in $37^{\circ} \mathrm{C}$ physiological saline solution.

The specimen was chucked with reference to the line markers on the frontal and medial surface of the femurACL-tibia complex with $0.5 \mathrm{~N}$ preloading. The extension rate was set at $0.01 \mathrm{~mm} / \mathrm{sec}$ and $200 \mathrm{~mm} / \mathrm{sec}$.

\section{RESULTS}

The measured strain-rate for each extension-rate condition of MCL substance and femur-ACL-tibia 
complex tensile tests are shown in Table 1 and 2, respectively. The extension rate was estimated as the cross-head velocity for quasi-static tests and the piston velocity for dynamic tests. The strain rate was calculated as the relative velocity between the two nygrosin markers.

Table 1. Extension-rate conditions and corresponding strain-rate conditions for ligament substance of MCL.

\begin{tabular}{cc}
\hline Extension Rate $(\mathrm{mm} / \mathrm{sec})$ & Strain Rate $(\% / \mathrm{sec})$ \\
\hline \hline 0.01 & $0.055 \pm 0.019$ \\
\hline 0.3 & $1.202 \pm 0.106$ \\
\hline 10 & $27.4 \pm 5.76$ \\
\hline $95.6 \pm 9.6$ & $653 \pm 118$ \\
\hline $278 \pm 27$ & $1007 \pm 256$ \\
\hline
\end{tabular}

(Mean \pm S.D.)

Table 2. Extension-rate conditions and corresponding strain-rate conditions for femur-ACL-tibia complex.

\begin{tabular}{cc}
\hline Extension Rate $(\mathrm{mm} / \mathrm{sec})$ & Strain Rate $(\% / \mathrm{sec})$ \\
\hline \hline 0.009 & $0.155 \pm 0.020$ \\
\hline $173.6 \pm 13.4$ & $2249 \pm 294$ \\
\hline & $($ Mean \pm S.D)
\end{tabular}

The stress-strain curves of the MCL substance with various strain-rate conditions are shown in Fig. 2. For the curves with 0.055 and $1.2 \% / \mathrm{sec}$ strain rate, we could see typical toe regions in low strain range and convex shape in the strain range over 5\%, however linear relations were observed in the stress-strain curves with $27.4,653$ and $1007 \%$ /sec strain rate.

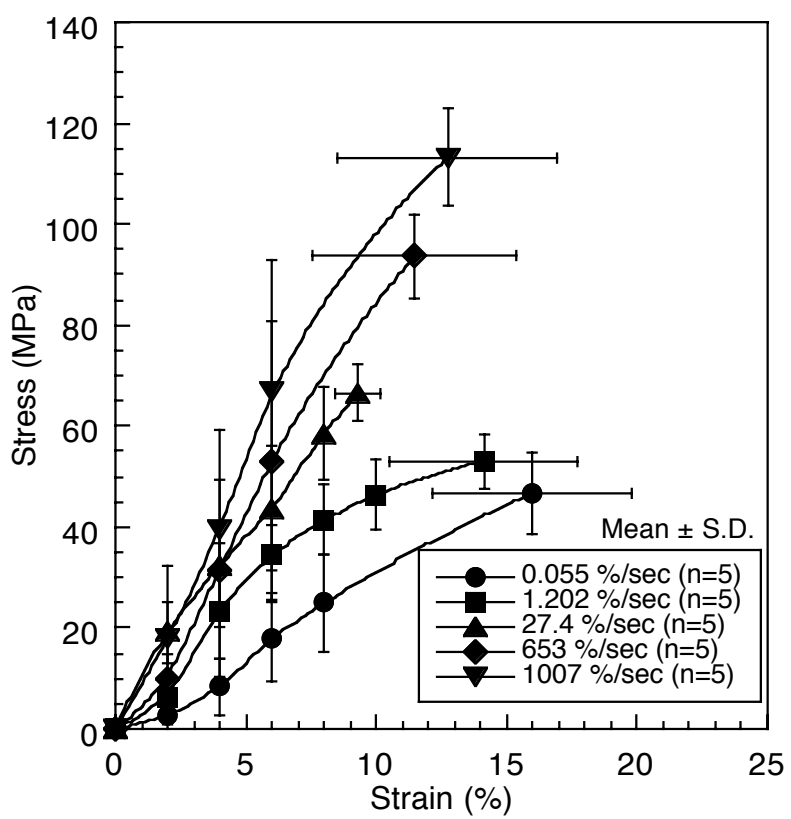

Fig. 2. Stress-strain relation of MCL ligament substance.

The tangent moduli of the stress-strain curves were examined in Fig. 3. They were defined as the moduli in the section between 3 to $7 \%$ for quasi-static tests and in the section between 2 to $6 \%$ for dynamic tests. The result of statistical analysis with one-way ANOVA did not show a significant difference among tangent moduli in each strain-rate condition $(\mathrm{p}>0.05)$. On the other hand, the failure stress increased with the strain rate and showed a statistically significant difference $(\mathrm{p}<0.05$, one-way ANOVA). This correlation is the same as that of the maximum stress of the femur-MCL-tibia complex ${ }^{[4]}$. In contrast, the failure strain did not show a significant difference among each of the strain-rate conditions ( $p>$ $0.05)$.

The failure loads of tibial insertion in each extensionrate condition are shown in Fig. 4, which also shows the failure loads of the MCL substance at various extensionrates. The failure load of the tibial insertion increased with the strain rate, and a statistically significant difference was observed with each experimental condition $(p<0.05)$. Thus, the strength of tibial insertion appears to depend on the strain rate just as with the failure stress of the MCL substance.

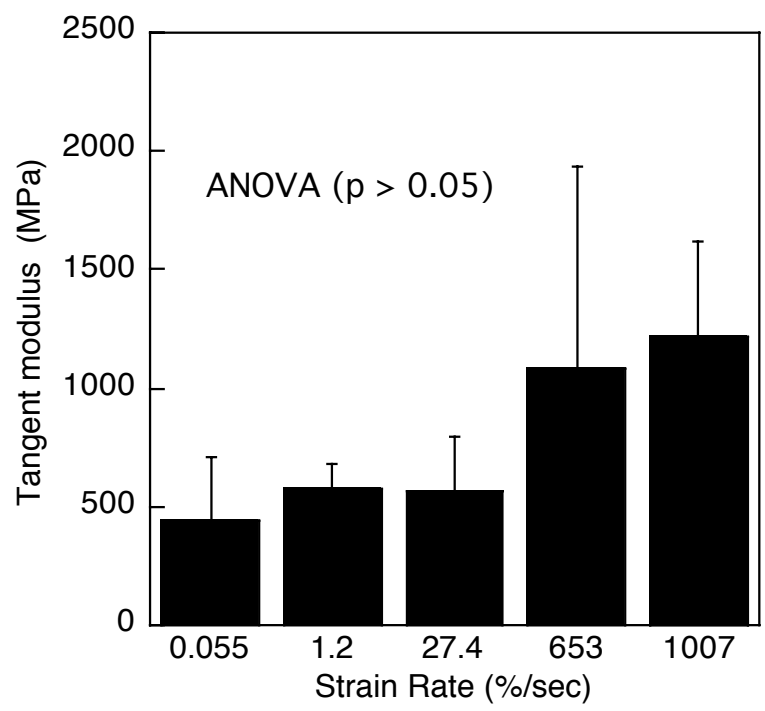

Fig. 3. Tangent moduli of stress-strain curve of MCL ligament substance.

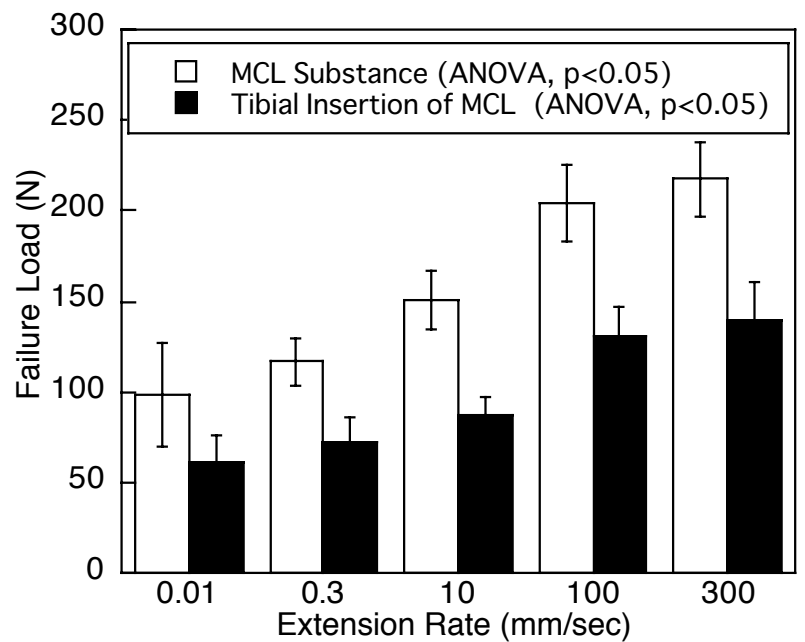

Fig. 4. Comparison between failure load of MCL tibial insertion and ligament substance at various strain rates. 
In the tensile tests of femur-ACL-tibia complex, three types of failure patterns were observed: avulsion fracture at femoral insertion, avulsion fracture at tibial insertion and epiphysiolysis of femoral distal condyle. These three failure patterns can be seen in traffic or sports accidents. The ratios of the frequency of each ACL failure pattern are shown in Fig. 5. In quasi-static tests, epiphysiolysis was the major failure pattern. On the other hand, the frequencies of every failure pattern were equal in the dynamic test condition.

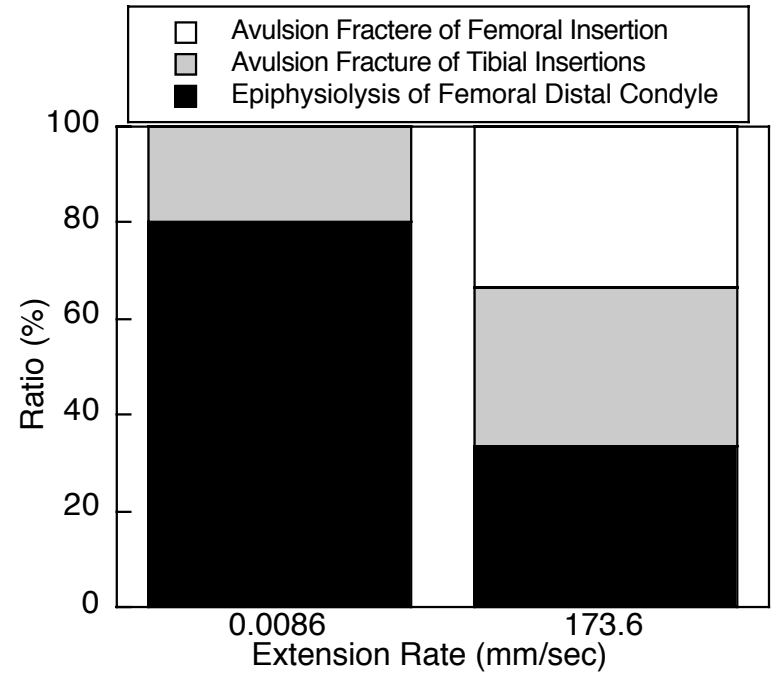

Fig. 5. Distributions of failure patterns of femur-ACLtibia complex.

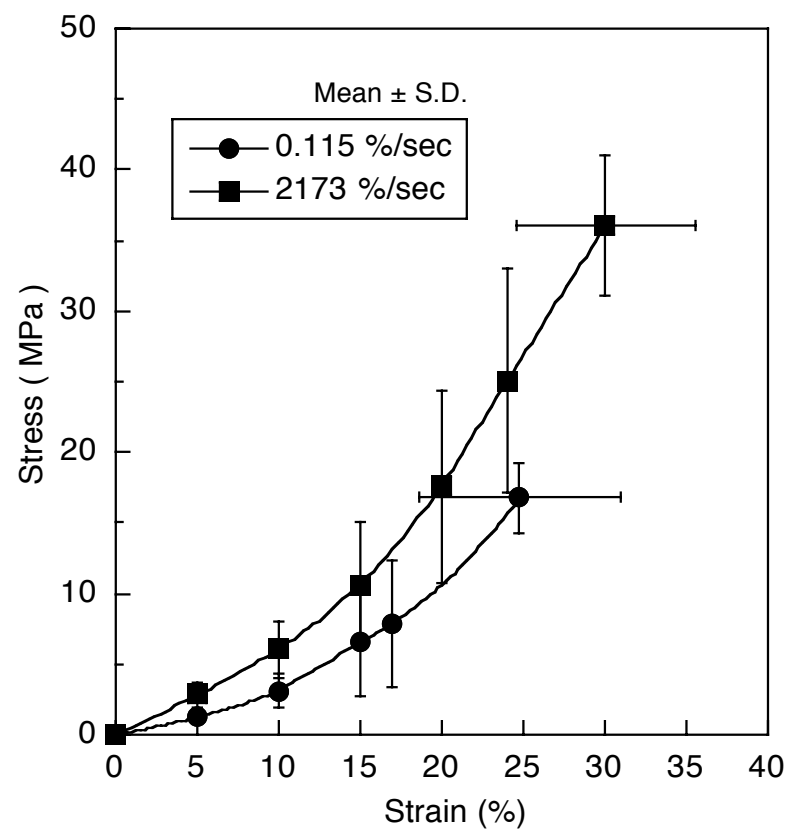

Fig. 6. Stress-strain curves of femur-ACL-tibia complex at various strain rates.

The stress-strain relation was evaluated based on the load-elongation relation and the cross-sectional area at the ACL mid-substance as shown in Fig. 6. With both strain rates, the stress-strain curves showed the nonlinear relation typically seen for biological soft tissue. The maximum stress tends to increase with the strain rate. It must be noted that these stress-strain relations do not indicate the exact mechanical properties of the ACL because the loading direction is not parallel to the longitudinal axis of the ACL. This figure indicates an apparent stress-strain relationship. To discuss the strainrate dependence of stress-strain relation, the tangent modulus was calculated in the $20-25 \%$ strain range for dynamic tests and in the $6-10 \mathrm{MPa}$ stress range for quasi-static tests. The tangent modulus for each condition was shown in Fig. 7. The tangent modulus showed a statistically significant difference in strain rate $(\mathrm{P}<0.05$, Student's $t$ test). The maximum stress and strain were also analyzed statistically. The maximum stress significantly increased with the strain rate $(\mathrm{P}<0.05$, Student's $t$ test) but the maximum strain did not depend on the strain rate $(\mathrm{P}>0.05$, Student's $t$ test).

\section{DISCUSSION}

The failure load of the tibial insertion is smaller than that of the MCL substance in all extension-rate conditions. This suggests that the tibial insertion is the most dangerous site in the femur-MCL-tibia region. However, an MCL rupture was observed at a high strainrate condition in our former study in which femur-MCLtibia complex used for a specimen [4]. In the present study, we removed the femoral insertion of the MCL in the preparation. That manipulation can cause loosening and inhomogenous tension of the collagen in the MCL, leading to possible underestimation of the failure load of the tibial insertion. In the experiments with the MCL substance, no fracture and avulsion were found in the femoral insertion of the MCL. The collagenous tissue of the MCL inserts into the medial condyle of femur and is tightly connected. On the other hand, the tibial end of the MCL is attached on the periosteum of the medial side of the proximal tibia. Such a structural characteristic causes a difference in the strength between the femoral insertion and the tibial insertion of the MCL. In the results of the cadaver test in which a car-to-pedestrian accident was simulated, an avulsion fracture of the femoral insertion of MCL was observed [6,7]. This type of injury was not observed in the current study. Thus, it is considered that such an avulsion fracture of the femoral insertion can be simulated by a lateral loading experiment. Experiments in which the loading condition in a real accident is considered are therefore needed.

The tangent modulus of the ACL was about twice of that of the MCL. Woo et al. [8] showed the tangent modulus of ACL is half as that of MCL in a quasicondition. To discuss these two different results the actual stress-strain relation of ACL must be evaluated.

Figure 7 shows the correlation between strain rate and failure stress in MCL substance or maximum stress of the femur-ACL-tibia complex. We obtained a logarithmic regression function with a high multiple correlation coefficient. This function shows the strain-rate dependence of the failure stress of the MCL substance and maximum stress of femur-ACL-tibia complex. In a comparison between the rate-dependence of the ACL and MCL, the coefficient of logarithmic regression function for the ACL was smaller than that of MCL.

The strain-rate dependence of the failure properties of the femur-ACL-tibia complex looks similar to those of the MCL substance. However, the maximum stress and 
strain discussed here may underestimate the failure properties of ACL because epiphysiolysis of the femoral distal condyle occurred with high frequency in this study. The cause of this high frequency could be the immaturity of the experimental animals. This disturbance may disappear with a more suitable experimental set up.

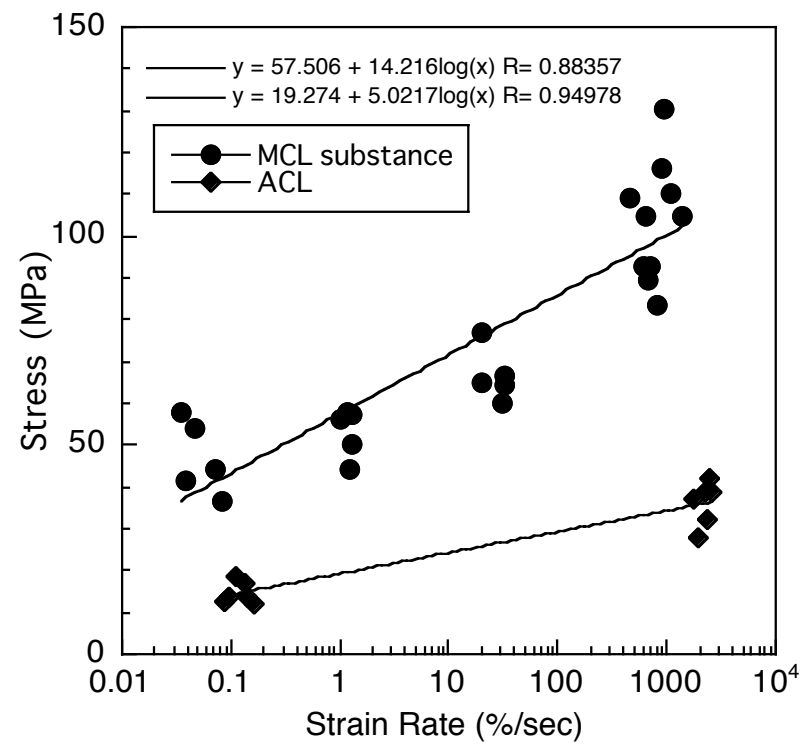

Fig. 7. Strain-rate dependence of failure stress of femur-ACL-tibia complex and MCL ligament substance.

No rupture in the ACL was observed in the tensile tests, unlike the case for traffic accidents. In a real-world accident, while axial tensile load is rarely applied, the shearing or bending load is likely to be applied. Therefore, other types of loading test, for example medial-lateral shearing or anterior drawer test, must be performed.

\section{CONCLUSIONS}

Mechanical properties of knee ligaments were discussed in the present study as a means to contribute to pedestrian protection. The strain-rate dependence of these properties was evaluated by quasi-static and dynamic tensile tests on the rabbit knee joint, and the following results were obtained.

(1) The failure stress of the MCL ligament substance significantly increased with the strain rate, but the failure strain and the tangent modulus did not depend on the strain rate.

(2) The failure load of the tibial insertion of the MCL is lower than that of the MCL ligament substance.

(3) Three types of failure, avulsion fracture at the femoral and tibial insertion of the ACL and the epiphysiolysis of femoral distal condyle, were observed in the tensile tests on the femur-ACL-tibia complex.

(4) The tangent modulus and maximum stress of the femur-ACL-tibia complex showed a significant strainrate dependence.

(5) No rupture in the ACL substance was observed. To simulate the actual loading conditions in real-world accidents, other types of loading test, for example a medial-lateral shearing or anterior drawer test, should be performed as the next step.

\section{REFERENCES}

1. Y. Takahashi, Y. Kikuchi et al., STAPP Car Crash Journal, 44 (2000), Paper No. 2000-01-SC22.

2. S.L-Y. Woo, R. H. Peterson et al., J Orthopaedic Res, 8-5 (1990), pp. 712-721.

3. N. Yamamoto, K. Hayashi, Bio-Medical Materials and Engin, 8 (1998), pp. 83-93.

4. S. Yamamoto, J. Kajzer et al., Human Biomechanics and Injury Prevention, ed. by Kajzer J, Tanaka E, Yamada H, Springer Verlarg Tokyo (2000), pp. 167172.

5. S.L-Y. Woo et al., J Biomechanics, 20-6 (1987), pp. 557-563.

6. J. Kajzer, G. Schroeder et al., SAE Paper No.973326, $41^{\text {st }}$ STAPP. (1997)

7. J. Kajzer, G. Schroeder et al., SAE Paper No. 199901-0712, Occupant Protection SAE SP-1432. (1999)

8. S.L-Y. Woo, O. Newton et al., J Biomechanics, 25-4 (1992), pp. 337-386. 